\title{
From genetic interactions to pathway architecture
}

DOI:

10.1038/nrg2052

URLs

MPH1

http://www.ncbi.nlm.nih. gov/entrez/query.fcgi?db=gen e\&cmd=Retrieve\&dopt=full_ report\&list_uids $=854818$ SGS1

http://www.ncbi.nlm.nih. gov/entrez/query.fcgi?db=gen e\&cmd=Retrieve\&dopt=full_ report\&list_uids $=855228$
Genome-wide screens for genetic interactions are an important tool for system-level analysis of cellular processes. Using fitness profiling of Saccharomyces cerevisiae deletion strains, St. Onge and colleagues have constructed a genetic interaction network that reveals novel insights into functional relationships and pathway architecture.

Theory predicts that deleterious effects of two alleles are multiplicative in a double mutant if the loci in question function independently. A departure from this expectation therefore identifies loci with products that interact functionally. St. Onge et al. used this test as the basis for identifying functional interactions among non-essential genes that are involved in resistance to chemically induced DNA damage.

The authors treated pools of homozygous deletion strains with a DNA-damaging agent, methyl methanesulfonate (MMS). They identified 26 deletion strains using chemogenomic fitness profiling. These were used to create all 650 pairwise combinations, and screens

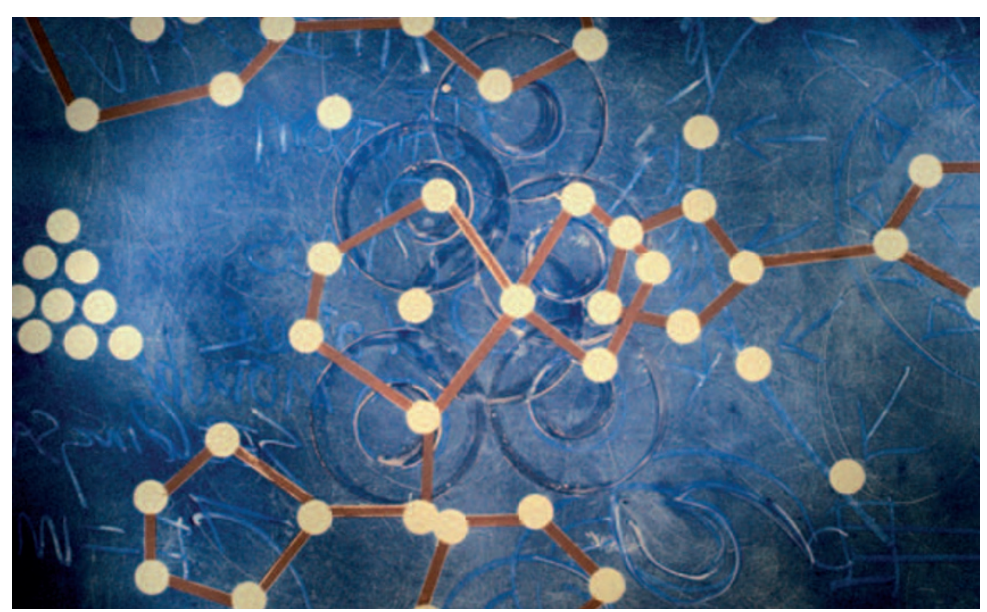

were carried out to identify deviations from the multiplicative model for fitness. The 'direction' of the deviation allowed the authors to differentiate between aggravating and alleviating interactions.

Focusing on a subset of gene pairs that they classified as alleviating in the presence of MMS, the authors subclassified these pairs to further define their relationships. There were cases of masking or partial masking epistasis, in which the effects of one deletion masked those of the other; complete and partial suppression, in which the effects of one deletion were reduced in the presence of the other; and 'co-equality', in which the effects of double and single deletions were statistically indistinguishable. Interactions that are similar to co-equality have been previously described as complementary epistasis or asynthetic relationships. As might be predicted, many co-equal partners encode physically interacting proteins. In these cases, deleting either gene product is sufficient to disrupt the function of the complex. 